\title{
Toxicité Aigüe Et Effets Des Extraits Des Feuilles De Cogniauxia Podolaena Bail. (Cucurbitaceae) Sur L'excrétion Fécale Et La Motilité Intestinale
}

\author{
Elion Itou $R D G$ \\ Etou Ossibi AW
}

Laboratoire de Biochimie et de Pharmacologie, Faculté des Sciences de la

Santé, Université Marien Ngouabi, Brazzaville-Congo

Laboratoire de physiologie animale, Faculté des Sciences et Techniques,

Université Marien Ngouabi, Brazzaville-Congo

\section{Morabandza CJ}

Laboratoire de Biochimie et de Pharmacologie, Faculté des Sciences de la

Santé, UniversitéMarien Ngouabi, Brazzaville-Congo

Département des Sciences Naturelles, École Normale Supérieure, Université

Marien Ngouabi Congo, Brazzaville - Congo

Kiessé D.S

Mayela Nkouka S.H.J

Abena AA

Laboratoire de Biochimie et de Pharmacologie, Faculté des Sciences de la

Santé, Université Marien Ngouabi, Brazzaville-Congo

doi: 10.19044/esj.2017.v13n27p355 URL:http://dx.doi.org/10.19044/esj.2017.v13n27p355

\begin{abstract}
This work aims to estimate acute toxicity and to evaluate the effects of the aqueous and hydroethanolic extracts of leaves (Cucurbitaceae) of Cogniauxia podolaena on the fecal excretion and the intestinal motility. The estimation of the acute toxicity was made by oral administration of the aqueous and hydroéthanolique extracts in the doses of 2000 and $500 \mathrm{mg} / \mathrm{kg}$ in mice in order to determine the lethal dose 50 (LD50) and the experimental doses. No sign of toxicity was observed with the hydroethanolic extract in mice. The aqueous extract of Cogniauxia podolaena is slightly toxic with a lethal dose estimated at the dose of $\quad 3167 \mathrm{mg} / \mathrm{kg}$. For that, the doses of 300 and $600 \mathrm{mg} / \mathrm{kg}$ were retained for experiment. The effect of the both extracts at 300 and $600 \mathrm{mg} / \mathrm{kg}$ was evaluated on the fecal excretion directly on the normal animals and on the intestinal motility according to the method of coal. The hydroethanolic extract (300 and $600 \mathrm{mg} / \mathrm{kg}$ ) increased the fecal excretion in rats. Aqueous extract (300 and $600 \mathrm{mg} / \mathrm{kg}$ ) did not present
\end{abstract}


effects. The both extracts in doses used does decrease the speed of intestinal evacuation. The effect of hydroethanolic extract is low at the dose of 600 $\mathrm{mg} / \mathrm{kg}$. These results do not seem to confirm the traditional use of this plant in the constipation coverage.

Keywords: Congo-traditional use-Acute toxicity-fecal excretion-intestinal motility-Cogniauxia podolaena

\section{Résumé}

Ce travail a pour objectif d'estimer la toxicité aigüe et d'évaluer les effets des extraits aqueux et hydroéthanolique des feuilles de Cogniauxia podolaena sur l'excrétion fécale et la motilité intestinale. L'estimation de la toxicité aigüe a été effectuée par administration orale des extraits aqueux et hydroéthanolique aux doses de 2000 et $500 \mathrm{mg} / \mathrm{g}$ chez la souris afin de déterminer la dose létale 50 (DL50) et les doses expérimentales. L'extrait hydroéthanolique n'a présenté aucun signe de toxicité chez les souris. L'extrait aqueux de Cogniauxia podolaena est faiblement toxique avec une dose létale estimée à la dose de $3167 \mathrm{mg} / \mathrm{kg}$. C'est ainsi que les doses de 300 et $600 \mathrm{mg} / \mathrm{kg}$ ont été retenues. L'effet des extraits à 300 et $600 \mathrm{mg} / \mathrm{k}$, a été évalué sur l'excrétion fécale directement sur les animaux normaux et sur la motilité intestinale selon la méthode de charbon. L'extrait hydroéthanolique aux doses de 300 et $600 \mathrm{mg} / \mathrm{kg}$ a augmenté l'excrétion fécale chez les rats. L'extrait aqueux aux mêmes doses n'a pas présenté d'effets. Les deux extraits aux doses utilisées diminuent la vitesse d'évacuation intestinale. L'effet de l'extrait hydroéthanolique est faible à la dose de $600 \mathrm{mg} / \mathrm{k}$. Ces résultats ne semblent confirmer l'usage traditionnel de la prise en charge de la constipation.

Mots-clés : Congo-Usage traditionnel-Toxicité aigüe, Excrétion fécale, Motilité intestinale, Cogniauxia podolaena

\section{Introduction}

Cogniauxia podolaena est une plante médicinale congolaise que l'on trouve aussi bien dans les savanes boisées du Pool ou des plateaux Batéké, que dans les forêts humides du Mayombe ou de la Cuvette congolaise [Bouquet, 1969]. Selon les enquêtes ethnobotaniques réalisées par Bouquet, (1969), la plante est un drastique extrêmement violent, éméto-cathartique ayant en plus une action diurétique et révulsive. Par contre, Adjanohoun et al, (1988) lui attribuent des propriétés analgésiques dans le traitement des douleurs abdominales. Les quelques études pharmacologiques réalisées au Congo ont démontré ses propriétés antipaludiques (Banzouzi et al., 2008), antalgique (Makambila et al., 2011) et contraceptive (Penemé et al., 2015). La plante contient dans les feuilles les alcaloïdes, les flavonoïdes et les 
hétérosides cardiotoniques (Kiéssé, 2016), la réserpine purgative et les terpènes-stéroïdes dans les racines (Kibungu Kembelo, 2004 ; Banzouzi et al, 2008). Cette étude vise à estimer la toxicité aigüe et d'évaluer les effets des extraits aqueux et hydroéthanolique des feuilles de Cogniauxia podolaena (Cucurbitaceae) sur l'excrétion fécale et la motilité intestinale afin de confirmer ou infirmer son usage traditionnel dans les troubles gastrointestinaux notamment dans la constipation.

\section{Matériel et méthodes}

\section{Matériel végétal}

Les feuilles sèches et pulvérisées de Cogniauxia podolaena (Cucurbitaceae) ont été utilisées. Ces feuilles ont été récoltées dans la forêt de l'Institut National de Recherche en Sciences Exactes et Naturelles (I.R.S.E.N) de Brazzaville en Mai 2014. L'identification a été réalisée par le Botaniste Systématicien le Docteur MOUTSAMBOTE. L'échantillon récolté a été comparé à un échantillon de référence $\left(n^{\circ} 7083\right)$ de l'herbarium de l'I.R.S.E .N. Le séchage des feuilles récoltées a été fait au Laboratoire de Biochimie et de Pharmacologie de la Faculté des Sciences de la Santé pendant 14 jours à la température de $27 \pm 1^{\circ} \mathrm{C}$. Après séchage, les feuilles ont été broyées à l'aide d'un mortier. La poudre obtenue a été conservée pour préparer les extraits aqueux et hydroéthanolique.

\section{Matériel animal}

Des rats albinos (Wistar) mâles et femelles de poids compris entre 150 et $250 \mathrm{~g}$ et des souris albinos (Swiss) mâles et femelles de poids variant entre 15 et $30 \mathrm{~g}$ ont été utilisés. Ces animaux ont été fournis par les animaleries de la Faculté des Sciences de la Santé et celle de la Faculté des Sciences et Techniques de 1'Université Marien Ngouabi (Congo). Ils ont été acclimatés pendant une semaine avant expérimentation et maintenus dans des conditions standards (12 heures d'éclairage, 12 heures d'obscurité) et à la température de $27 \pm 1^{\circ} \mathrm{C}$. Ils ont été mis à jeun 24 heures avant expérimentation. L'eau de robinet leur était donnée à volonté.

\section{Méthodes}

\section{Préparation des extraits}

L'extrait aqueux a été préparé par décoction. $20 \mathrm{~g}$ de poudre de Cogniauxia podolaena ont été mis en ébullition dans $200 \mathrm{ml}$ d'eau distillée pendant 15 minutes. Après refroidissement puis filtration, le décocté obtenu a été concentré au bain marie $\left(60^{\circ} \mathrm{C}\right)$. L'extrait aqueux obtenu a été conservé pour les expérimentations. L'extrait hydroéthanolique a été préparé par macération. $25 \mathrm{~g}$ de poudre de Cogniauxia podolaena ont été mélangés dans $250 \mathrm{ml}$ d'éthanol à $70 \%$. L'ensemble a été mis sous agitation 
magnétique pendant 24 heures. Le macéré obtenu après filtration a été concentré à l'aide d'un bain marie $\left(60^{\circ} \mathrm{C}\right)$.

\section{Toxicité aigüe}

La toxicité aigüe a été estimée conformément à la ligne directrice $\mathrm{n}^{\circ} 423$ de l'OCDE, (2001).

Les souris ont été mises à jeun pendant 24 heures avant l'administration orale des produits. Cinq lots de 3 souris chacun ont été constitués et traités comme suit: lot1 témoin traité avec de l'eau distillée $(0,5 \mathrm{ml} / 100 \mathrm{~g})$, lots 2 et 3 traités avec l'extrait aqueux aux doses respectives 2000 et $5000 \mathrm{mg} / \mathrm{kg}$, lots 4 et 5 traités avec l'extrait hydroéthanolique aux doses respectives de 2000 et $5000 \mathrm{mg} / \mathrm{kg}$. Les observations ont été faites toutes les trente minutes durant 4 heures. Elles ont portées sur le ptosis, la piloérection, l'agressivité, la mobilité, la vigilance, l'état des selles, le vomissement et la mortalité. La mortalité a été évaluée pendant 48 heures. Au cas où la mort des souris était observée, la dose qui tue la moitié des animaux (DL50) a été déterminée par la formule suivante :

DL50 = DmM $-\frac{\sum(\mathbf{a X b}) \mathbf{x}}{\mathbf{n}}$ [Chippaux et al., 1997]

DmM : dose minimale mortelle, c'est la plus faible dose qui tue tous les animaux.

a: différence entre deux doses consécutives ; b : moyenne de morts entre deux doses consécutives ; $\mathrm{n}$ : nombre d'animaux par lot.

Évaluation de l'effet des extraits aqueux et hydroéthanolique de Cogniauxia podolaena sur l'excrétion fécale

Cet effet a été évalué en utilisant la méthode décrite par Mengyi et al., (2013). Six (6) lots de cinq (5) rats chacun ont été constitués et traités de la manière suivante : lot 1traité avec de l'eau distillée (Témoin : 0,5 $\mathrm{ml} / 100 \mathrm{~g}$ ), lot 2 traité avec de l'huile de ricin (Molécule standard : $2 \mathrm{ml} / \mathrm{rat}$ ), lots 3 et 4 traités avec l'extrait aqueux de Cogniauxia podolaena (300 et 600 $\mathrm{mg} / \mathrm{kg}$ ), lots 5 et 6 traités avec l'extrait hydroéthanolique de Cogniauxia podolaena (300 et $600 \mathrm{mg} / \mathrm{kg}$ ). Après administration orale de ces produits, les animaux ont été placés dans des cages à métabolisme pour observation. La quantité et la fréquence d'émission des selles ont été évaluées pendant 8 heures. L'effet sur l'excrétion fécale est donné par le pourcentage de production des selles (PS) :

PS

$=\frac{\text { Quantité des selles émises par les animaux traités }- \text { Quantité des selles émises par les animaux témoins }}{}$

$\times 100$

Quantité des selles émises par les animaux traités

Évaluation de l'effet des extraits aqueux et hydroéthanolique de Cogniauxia podolaena sur la motilité intestinale 
Cet effet a été évalué en utilisant la méthode de charbon [Elion Itou, 2010]. Pour cela, six (6) lots de cinq (5) rats chacun ont été constitués. L'eau distillée (témoin, $0,5 \mathrm{ml} / 100 \mathrm{~g}$ ), le lopéramide (médicament de référence, 10 $\mathrm{mg} / \mathrm{kg}$ ), l'extrait aqueux de Cogniauxia podolaena $(300$ et $600 \mathrm{mg} / \mathrm{kg}$ ) et l'extrait hydroéthanolique de Cogniauxia podolaena (300 et $600 \mathrm{mg} / \mathrm{kg}$ ) ont été administrés par voie arole chez les rats une (1) heure avant administration du charbon $10 \%$ (Norit*, $10 \mathrm{ml} / \mathrm{kg}$ per os) utilisé comme marqueur. $30 \mathrm{mn}$ après, les animaux ont été sacrifiés par dislocation cervicale, l'abdomen ouvert, l'intestin grêle prélevé et posé sur du papier buvard. La distance parcourue par le charbon est alors mesurée à l'aide d'une règle graduée et exprimée en pourcentage $(\%)$ du transit intestinal :

$$
\text { Transit intestinal }=\frac{\text { distance parcourue par le charbon }}{\text { longueur totale de l'intestin grele }} \times 100
$$

Évaluation de l'effet de l'extrait hydroéthanolique des feuilles de Cogniauxia podolaena sur l'excrétion fécale en présence de l'huile de ricin

Cet effet a été évalué en utilisant l'huile de ricin comme laxatif de référence. Cinq (05) lots de cinq (5) rats chacun et traités de la manière suivante : L'eau distillée $(0,5 \mathrm{ml} / 100 \mathrm{~g})$, huile de ricin plus $(+)$ l'eau distillée (témoin), l'huile de ricin plus $(+)$ le lopéramide (antidiarrhéique de référence, $10 \mathrm{mg} / \mathrm{kg})$, l'huile de ricin plus (+) l'extrait l'hydroéthanolique de Cogniauxia podolaena (300 et $600 \mathrm{mg} / \mathrm{kg}$ ). L'eau distillée, le lopéramide et l'extrait l'hydroéthanolique de Cogniauxia podolaena ont été administrés une heure après administration de l'huile de ricin. Ainsi, une (1) heure après administration orale de ces produits, la fréquence d'émission des selles ainsi que la somme des selles excrétées avant et après administration des produits ont été notées pendant 8 heures. L'effet sur l'excrétion fécale a été évalué par comparaison avec le groupe témoin et exprimé en pourcentage de production des selles.

\section{Analyse statistique des résultats}

Les résultats sont exprimés en moyenne plus ou moins $( \pm)$ erreur standard sur la moyenne. Les résultats obtenus dans les groupes tests ont été comparés au groupe témoin traité avec l'eau distillée. Le test $« \mathrm{t} »$ de Student a été utilisé et le seuil de significativité fixé à $\mathrm{p}<0,05$.

\section{Résultats}

\section{Toxicité aigüe}

L'extrait aqueux de Cogniauxia podolaena administré par voie orale aux doses de 2000 et $5000 \mathrm{mg} / \mathrm{kg}$ provoque une diminution de la mobilité, de la sensibilité à la douleur induite par le pincement de la queue ainsi que la réaction aux stimuli extérieurs par rapport aux souris témoins. Les taux de 
mortalité sont de 33,33 et $100 \%$ aux doses respectives de 2000 et 5000 $\mathrm{mg} / \mathrm{kg}$. La dose létale 50 est estimée à la dose de à $3167 \mathrm{mg} / \mathrm{kg}$. Cependant, ni modification de l'état général, ni mortalité n'ont été observée avec l'extrait hydroéthanolique aux doses utilisées.

Effets des extraits aqueux et hydroéthanolique de Cogniauxia podolaena sur l'excrétion fécale chez le rat normal

Les résultats des effets des extraits aqueux et hydroéthanolique de Cogniauxia podolaena sur l'excrétion fécale sont présentés sur les figures 1, 2 et 3 . Ces figures montrent que les animaux traités avec le laxatif de référence, l'huile de ricin ainsi que ceux traités avec l'extrait hydroéthanolique $(300$ et $600 \mathrm{mg} / \mathrm{kg})$ ont émis de selles en quantité importante $(\mathrm{p}<0,01 ; \mathrm{p}<0,001)$ avec des fréquences élevées $(\mathrm{p}<0,05$; $\mathrm{p}<0,01$ ) comparativement au groupe contrôle eau distillée (figure 1 et 3 ). La production de selles est de $96,66 \%$ avec l'huile de ricin, de 81,14 et $81,7 \%$ pour l'extrait hydroéthanolique aux doses respectives de 300 et $600 \mathrm{mg} / \mathrm{kg}$ (figure 2). La production des selles obtenue avec l'extrait hydroéthanolique est largement inférieure à celle obtenue avec l'huile de ricin (figures 2). Cependant, la production des selles est de 0 et $32,35 \%$ et la fréquence de 0 et 0,4 fois pendant 8 heures avec l'extrait aqueux de Cogniauxia podolaena aux doses de 300 et $600 \mathrm{mg} / \mathrm{kg}$ (figures 1,2 et 3 ). intestinale

Effet des extraits aqueux et hydroéthanolique sur la motilité

Les effets des extraits aqueux et hydroéthanolique de Cogniauxia podolaena sur la motilité intestinale sont sur la figure 4. Aux doses utilisées, l'extrait aqueux (300 et $600 \mathrm{mg} / \mathrm{kg}$ ) et l'extrait hydroéthanolique (300 $\mathrm{mg} / \mathrm{kg}$ ) n'ont pas augmenté la vitesse de propulsion du charbon par rapport au groupe contrôle. Au contraire, ils réduisent significativement comme le lopéramide (molécule de référence) la vitesse d'évacuation du contenu intestinal. Cependant à la dose de $600 \mathrm{mg} / \mathrm{kg} \mathrm{l'extrait} \mathrm{hydroéthanolique} \mathrm{est}$ sans effets sur la vitesse d'évacuation gastrique par comparaison au groupe contrôle eau distillée (figure 4)

\section{Effet de l'extrait hydroéthanolique sur l'excrétion fécale en présence de l'huile de ricin}

Les résultats de l'effet de l'extrait hydroéthanolique sur l'excrétion fécale induite par l'huile de ricin sont présentés sur les figures 5, 6 et 7. Ils montrent que les animaux qui ont reçu de l'huile de ricin seule ont excrété beaucoup de selles par rapport à ceux qui n'ont reçu que de l'eau distillée (figures 5 et 7). Cependant, l'extrait hydroéthanolique à la dose de 300 $\mathrm{mg} / \mathrm{kg}$ réduit significativement comme le lopéramide (antidiarrhéique de référence) la quantité et la fréquence des selles par rapport au témoin eau distillée (figures 5 et 7). Ceci s'est traduit par une inhibition importante de la 
production des selles respectivement de 61,82 et 59,36 \% pour l'extrait hydroéthanolique $(300 \mathrm{mg} / \mathrm{kg}$ ) et le lopéramide (figure 6). Par contre, on note aussi une réduction significative de la quantité et la fréquence d'émission des selles par rapport au témoin eau distillée avec l'extrait hydroéthanolique à la dose de $600 \mathrm{mg} / \mathrm{kg}$. Cette inhibition $(39,91 \%)$ est nettement inférieure à celle observée avec le même extrait à la dose de $300 \mathrm{mg} / \mathrm{kg}$ et le lopéramide (figure 6).

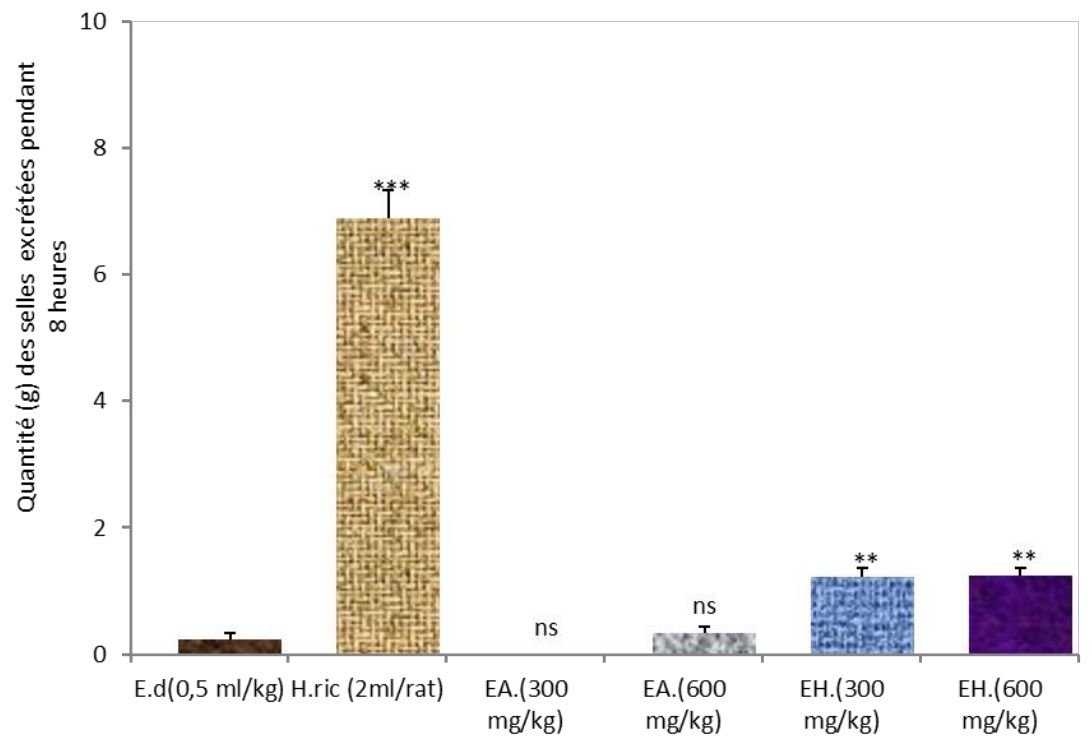

Figure 1: Effet des extraits aqueux (EA) et hydroéthanolique (EH) de Cogniauxia podolaena sur la quantité de selles excrétées. Chaque valeur représente la moyenne de la quantité (Q) des selles plus ou moins erreur Standard. $\mathrm{Q} \pm \mathrm{ESM} ; * * \mathrm{p}<0,01$ différence significative par rapport au témoin eau distillée (E.d). ns : différence non significative par rapport au témoin. H.ric=Huile de ricin ; lop=lopéramide

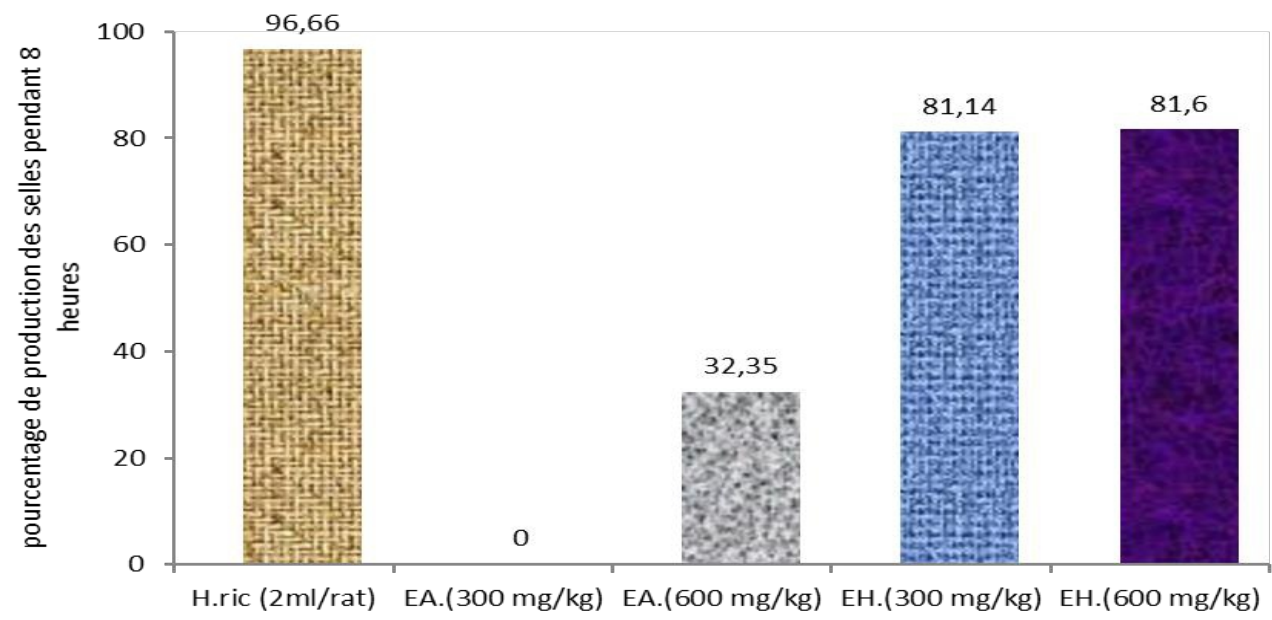

Figure 2 : Pourcentage de production des selles des extraits aqueux aqueux (EA) et hydroéthanolique $(\mathrm{EH})$ de Cogniauxia podolaena sur l'excrétion fécale. H.ric=huile de ricin 


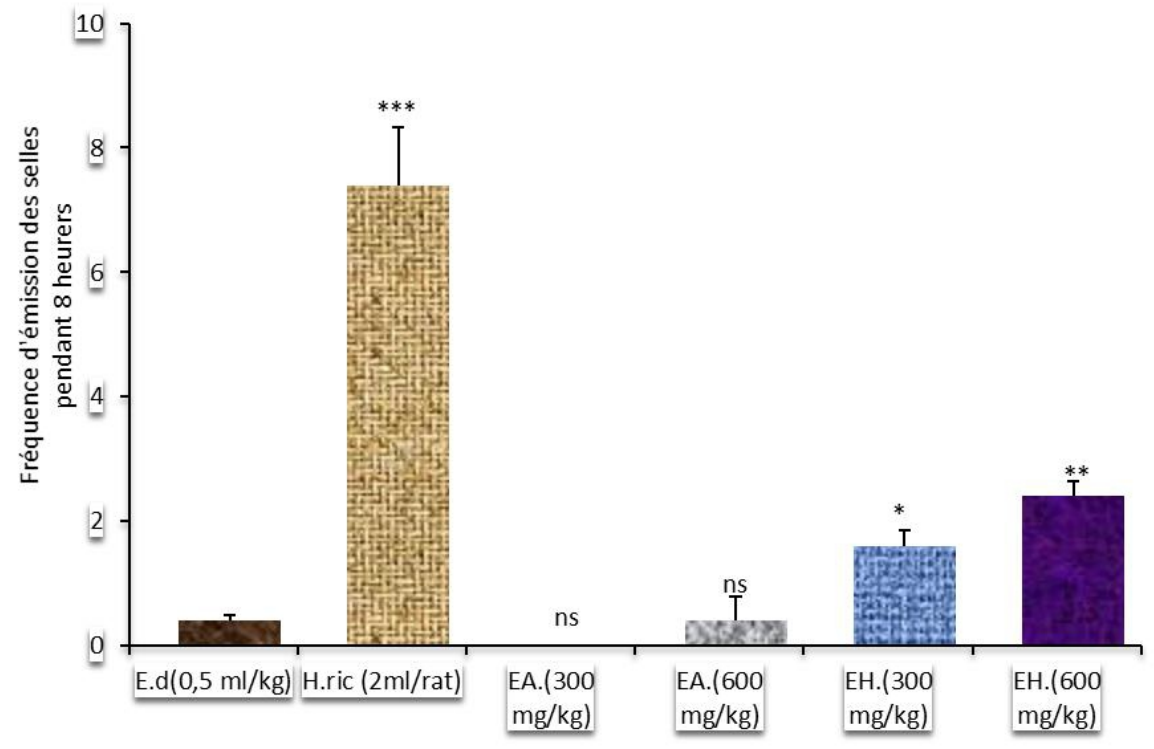

Figure 3: Effet de l'extrait aqueux (EA) et hydroéthanolique (EH) de Cogniauxia podolaena sur la fréquence d'excrétion fécale. Chaque valeur représente la moyenne de la fréquence

(F) d'excrétion fécale plus ou moins erreur Standard. Q \pm ESM ; ${ }^{*} p<0,05$ et $* * p<0,01$ différences significatives par rapport au témoin eau distillée (E.d). ns=différence non significative par rapport au témoin. H.ric=huile de ricin ; lop=lopéramide

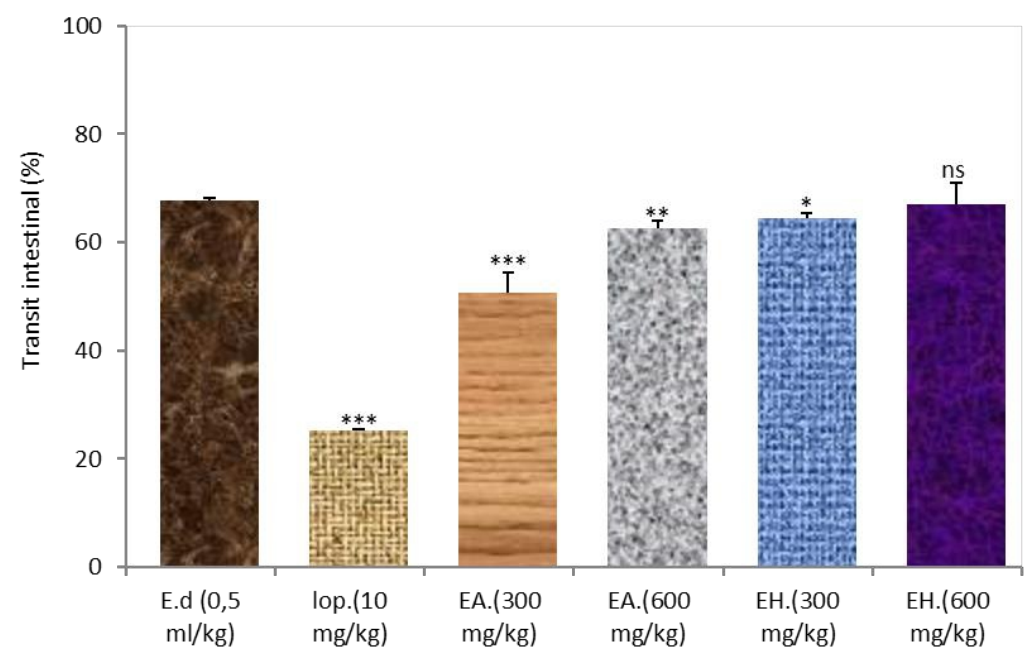

Figure 4: Effet de l'extrait aqueux (EA) et hydroéthanolique (EH) de Cogniauxia podolaena sur le transit intestinal. Chaque valeur représente la moyenne du transit $(\mathrm{T})$ plus ou moins erreur standard. T $\pm \mathrm{ESM} ; * \mathrm{p}<0,05 ; * * \mathrm{p}<0,01 ; * * * \mathrm{P}<0,001 ;$ test significatif par rapport au groupe témoin eau distillée. ns : valeur non significative par rapport au groupe contrôle eau distillée. 


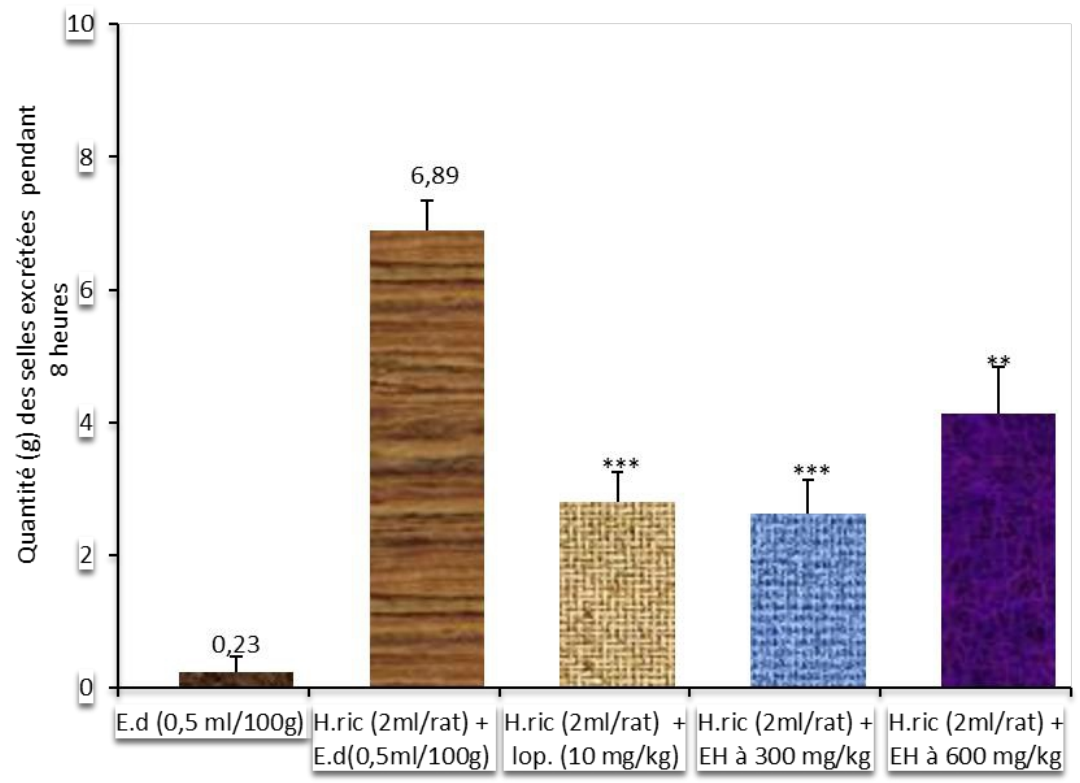

Figure 5: Effet de l'extrait hydroéthanolique (EH) de Cogniauxia podolaena sur la quantité des selles en présence de l'huile de ricin (H.ric). Chaque valeur représente la moyenne de la quantité (Q) des selles plus ou moins erreur standard. Q $\pm \mathrm{ESM}$; $* * * \mathrm{P}<0,001 ; * * \mathrm{p}<0,01$ test de Student significatif par rapport au témoin (H.ric seule)). E. $d=$ eau distillée ; lop= lopéramide

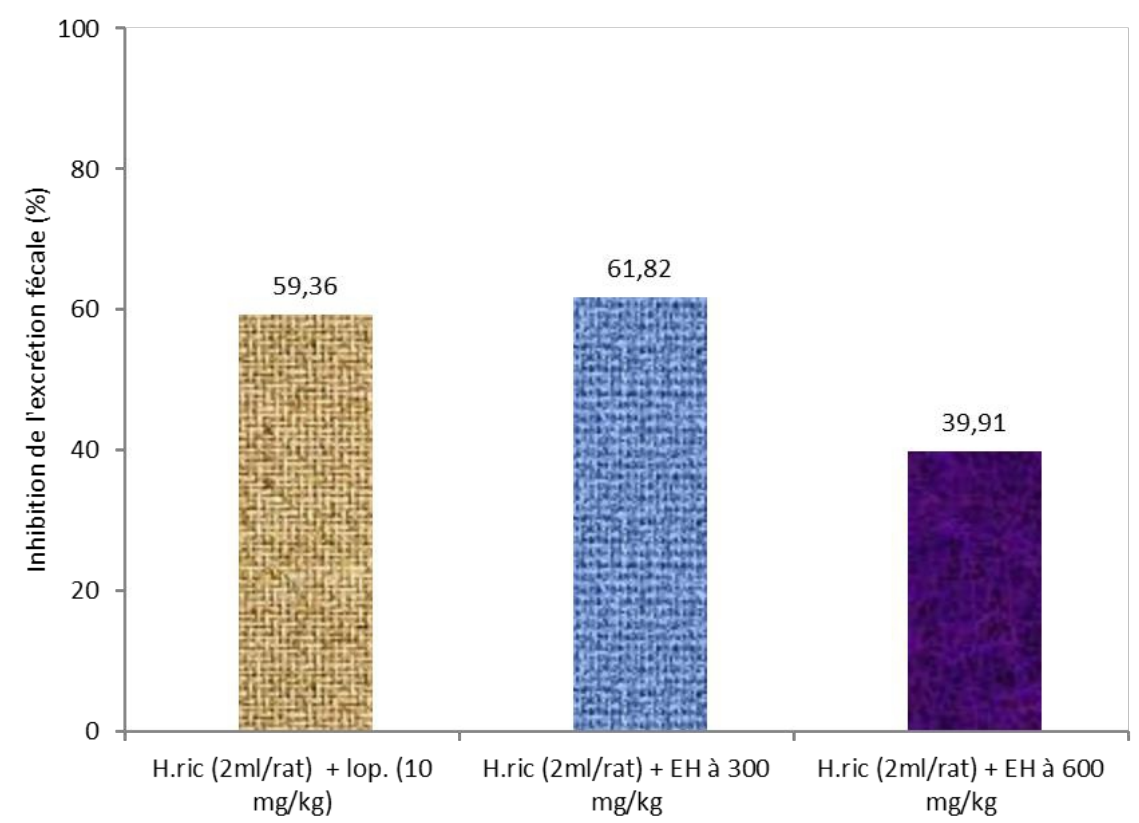

Figure 6: Inhibition de l'excrétion fécale par l'extrait hydroéthanolique (EH) de Cogniauxia podolaena en présence de l'huile de ricin (H.ric). lop= lopéramide 


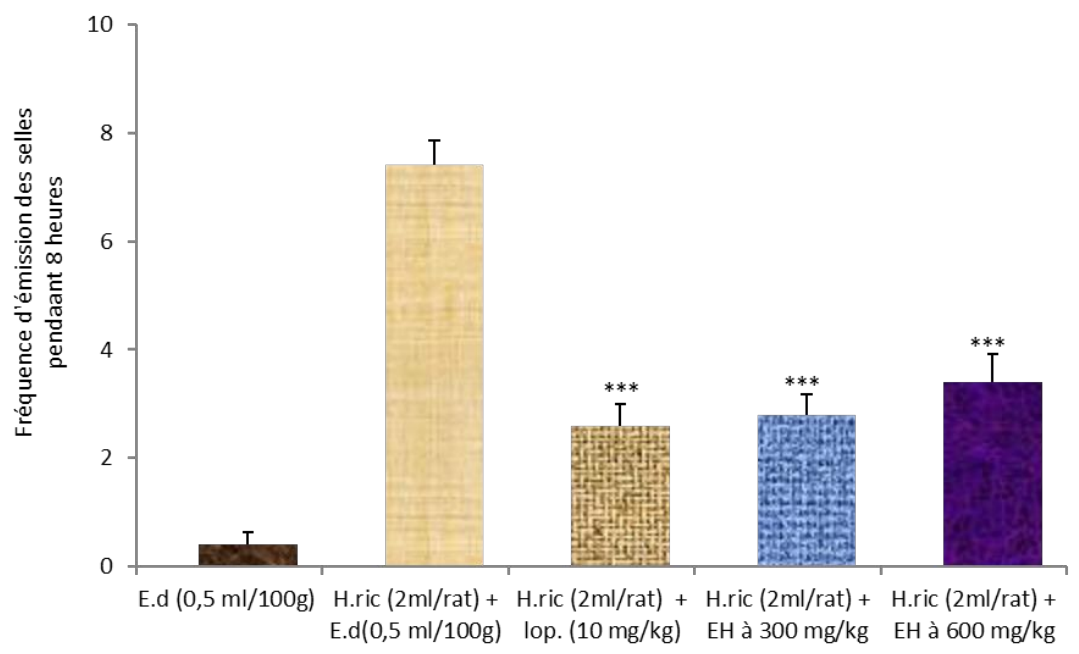

Figure 7: Effet de l'extrait hydroéthanolique (EH) de Cogniauxia podolaena sur la fréquence d'émission des selles en présence de l'huile de ricin (H.ric). Chaque valeur représente la moyenne de la fréquence $(\mathrm{F})$ des selles plus ou moins erreur standard. $\mathrm{F} \pm$ $\mathrm{ESM} ; * * * \mathrm{P}<0,001$; test de Student significatif par rapport au témoin (H.ric seule). $\mathrm{E} . \mathrm{d}=$ eau distillée ; lop= lopéramide

\section{Discussion}

Avant d'évaluer les effets des extraits aqueux et hydroéthanolique de Cogniauxia podolaena sur l'excrétion fécale et la motilité intestinale, nous avons estimé leur toxicité aigüe chez la souris afin de déterminer la dose létale 50 (DL50) et les doses expérimentales. L'extrait aqueux des feuilles de Cogniauxia podolaena en administration orale unique provoque la diminution de la mobilité et entraine une somnolence mettant les animaux dans un état de fatigue générale. Ce résultat suggère des probables effets sédatifs de l'extrait aqueux des feuilles de Cogniauxia podolaena. Cependant, ni modification de l'état général, ni mortalité des animaux n'ont été observées avec l'extrait hydroéthanolique. Par ailleurs, les taux de mortalité sont de 33,33 et $100 \%$ aux doses respectives de 2000 et 5000 $\mathrm{mg} / \mathrm{kg}$ de l'extrait aqueux des feuilles de Cogniauxia podolaena. La dose létale 50 est estimée à la dose de $3167 \mathrm{mg} / \mathrm{kg}$. Ce résultat suggère que l'extrait aqueux des feuilles de Cogniauxia podolaena est faiblement toxique. Selon le système de classification globalement harmonisé, Cogniauxia podolaena serait classé dans la catégorie 5 des plantes présentant un danger pour l'organisme [OCDE, 2001]. La DL50 de l'extrait aqueux des feuilles de Cogniauxia podolaena étant de $3167 \mathrm{mg} / \mathrm{kg}$, les doses correspondant au dixième $(300 \mathrm{mg} / \mathrm{kg})$ et au cinquième $(600 \mathrm{mg} / \mathrm{kg})$ ont été retenues pour évaluer les effets sur l'excrétion fécale et la motilité intestinale. L'extrait hydroéthanolique de Cogniauxia podolaena aux doses 
de 300 et $600 \mathrm{mg} / \mathrm{kg}$ a augmenté la production des selles de manière significative par rapport au groupe contrôle eau distillée. Ces effets sont inférieurs à ceux de l'huile de ricin utilisée comme laxatif de référence. En effet, cette huile possède un principe actif nommé acide ricinoléique doté d'un pouvoir purgatif [Bruneton, 1993]. L'acide ricinoleique libéré produit des changements dans le transport de l'eau et des électrolytes avec pour résultat une hypersécrétion et une augmentation de la vitesse du transit intestinal [Lenika et al., 2005]. Plusieurs facteurs interviennent dans la physiologie intestinale pour expliquer la production des selles ou une augmentation de la fréquence de leur production. Il pourrait s'agir d'une production d'eau au niveau du colon ou d'un effet directe du produit sur la matière fécale en la ramollissant comme le ferait certains laxatifs dits « ramollissant" ou en rendant les selles grasses comme les laxatifs lubrifiants. Le fait que l'extrait hydroéthanolique aux doses de 300 et 600 $\mathrm{mg} / \mathrm{kg}$ de Cogniauxia podolaena augmente la quantité et la fréquence des selles suggère qu'il pourrait interférer avec les mécanismes d'excrétion des électrolytes. La perturbation des mécanismes d'excrétion des électrolytes au niveau de l'intestin et l'effet direct des produits sur la matière fécale ne sont pas les seuls à expliquer l'augmentation de la fréquence donc la quantité des selles produites. Ceci pourrait être aussi dû à un effet sur la vitesse d'évacuation intestinale. C'est pourquoi l'effet sur la motilité intestinale a été évalué. Les extraits aqueux aux doses de 300 et $600 \mathrm{mg} / \mathrm{kg}$ et hydroéthanolique de Cogniauxia podolaena à la dose de $300 \mathrm{mg} / \mathrm{kg}$ ont réduit significativement la vitesse d'évacuation du contenu intestinal comme le lopéramide (antidiarrhéique de référence). Ces résultats suggèrent que les effets des extraits aqueux et hydroéthanolique observés sur l'excrétion fécale pourraient être dûs à une interférence avec le système nerveux autonome qui contrôle la motilité intestinale. Pour chercher à comprendre les effets de l'extrait hydroéthanolique sur l'excrétion fécale et la motilité intestinale, nous avons évalué son effet sur l'excrétion fécale chez les animaux prétraités par de l'huile de ricin. Les résultats montrent que le lopéramide et l'extrait hydroéthanolique aux doses utilisées s'opposent aux effets laxatifs de l'huile de ricin. Par contre, la dose de $600 \mathrm{mg} / \mathrm{kg}$ a produit plus de selles que celle de $300 \mathrm{mg} / \mathrm{kg}$. Ces résultats suggèrent que l'extrait hydroéthanolique n'agirait pas comme l'huile de ricin utilisée comme laxatif de référence, donc ne stimulerait pas l'excrétion fécale.

\section{Conclusion}

Cogniauxia podolaena est une plante médicine congolaise utilisée dans le traitement des troubles gastro-intestinaux. L'extrait aqueux de Cogniauxia podolaena est faiblement toxique avec une dose létale 50 égale à $3167 \mathrm{mg} / \mathrm{kg}$ alors que l'extrait hydroéthanolique est non toxique jusqu'à la 
dose $5000 \mathrm{mg} / \mathrm{kg}$. L'extrait aqueux (300 et $600 \mathrm{mg} / \mathrm{kg}$ ) n'augmente pas l'excrétion fécale contrairement à l'extrait hydroéthanolique (300 et 600 $\mathrm{mg} / \mathrm{kg}$ ). Cependant, ces deux extraits ne seraient pas laxatifs. Cette étude mérite d'être approfondie avec les autres parties de la plante afin de clarifier son usage traditionnel.

\section{References:}

1. Adjanohoum E., Ahyi A.M.A., Aké Assi L., J Baniakina., P Chibon., G Cusset., V Doulou., A Enzanza., J Eymé., E Goudoté., A Keita., C Mbemba, J Mollet., J.M Moutsamboté., J Mpati et P Sita. Médecine traditionnelle et pharmacopée. Contribution aux études ethnobotaniques et floristiques en République Populaire du Congo, Edition ACCT, Paris : 605p, 1988

2. Banzouzi JT., Soh PN., Mbatchi B., Cavé A., Ramos S., Retailleau P., Rakotonandrasana O., Berry A, Benoit-Vical F.Cogniauxia podolaena: bioassay-guided fractionation of defoliated stems, isolation of active compounds antiplasmodial activity and cytotoxicity. Planta Med.; 74 (12):1453-6, 2008

3. Bouquet A. Féticheurs et médecines traditionnelles du Congo (Brazzaville). O.R.S.T.O.M, $36: 282 p, 1969$.

4. Bruneton Jean . Pharmacognosie ; Phytochimie Plantes médicinales 2 ème éd Technique et Documentation-Lavoisièr : 760-761, 1993

5. Chippaux J.P., Rakotoria V.S., Rakotoria A., Dzikouk C. Substances médicamenteuses antagonistes du venin ou potentialisant le sérum antivenimeux ; Bulletin de la société de pathologie exotique, 90 : 282-285, 1997

6. Elion Itou R.DG. Effets antiulcéreux et antidiarrhéique de Ceiba Pentandra Gaertn (Bombacaceae).Thèse de doctorat unique de l'Université Marien Ngouabi, Brazzaville-Congo : 158, 2010

7. Kibungu Kembelo A.O. Quelques plantes médicinales du Bas-Congo et leurs usages, DFID, London, United Kingdom : 195p, 2004

8. Kiessé D.S. Toxicité aigüe et effets des extraits aqueux et hydroéthanolique des feuilles de Cogniauxia podolaena (Cucurbitaceae) bail sur la motilité intestinale et l'excrétion fécale chez le rat wistar, Mémoire de Master, Faculté des Sciences et Techniques-Université Marien Ngouabi- Brazzaville-Congo : 39p, 2016

9. Lenika S., Rajesh S., and S Ojha. Evaluation of antimotility effect of Lantana Camara L.Var. acuelata Constituents on neostigmine induced gastrointestinal transit in mice, BMC Complement Altern Med; 5:18, 2005 
10. Makambila-Kobemba M.Cl., B Mbatchi., D Ardid., A Gelot., C Henrison., R Janisson., AA Abena and JT Banzouzi. Pharmacological Studies of ten Medicinal plants used for analgesic purposes in Congo Brazzaville. International journal of pharmacology. 7: 608-615, 2011

11. OCDE. Harmonized Integrated Hazard Classification system for Human Health and Environmental Effects of Chemical substances, part 2, OECD Paris: 20-24, 2001

12. Penemé M.B., Andissa N.O., Mouankié J.B., Binimbi Massengo., Arnaud Wilfrid Etou Ossibi et Ange Antoine ABENA. Effet contraceptif de l'extrait aqueux des feuilles de Cogniauxia podolaena et de Buchhozia coriacea sur la fécondité chez la ratte de type wistar, Afrique Science, 11(4) : 131-140, 2015

13. Mengyi Z., Ping J., Jinping C., Aihui X., Yue Z., Yibei Z., Peng C and Jianfa $Z$. Laxative effects of Salecan on normal and two models of experimental constipated mice, BMC Gastroenterology ; 13: 52, 2013 